Bull. Austral. Math. Soc.

VOL. $71(2005)$ [215-224]

\title{
PROXIMAL PROPER EFFICIENCY FOR MINIMISATION WITH RESPECT TO NORMAL CONES
}

\author{
C.S. Lalitha and Ruchi Arora
}

This paper is devoted to the study of a new kind of proper efficiency in terms of proximal normal cones for vector minimisation. This new notion called proximal proper efficiency is used to obtain a scalar characterisation when a set related to the criterion set is a nonconvex set. Proximal proper efficiency is related with the well known notions of Benson and Borwein proper efficiency which are defined in the literature in terms of tangent cones. The study is further extended to characterise Benson and Borwein proper efficiency in terms of normal cones assuming convexity of a related set.

\section{INTRODUCTION}

The presence of an objective function with more than one criterion is a very common feature in many decision making problems. The concept of optimal solutions to such a multiobjective optimisation problem is not as trivial as in the case of a single objective optimisation problem. It is usually related to the preference attitudes of the decision makers. One of the most fundamental concepts is that of efficient solution or nondominated solution.

Kuhn-Tucker [9] observed that not every efficient point can be characterised by a scalar optimisation problem even if the decision set is convex. In order to avoid such undesirable efficient solutions Kuhn-Tucker [9] introduced the notion of proper efficiency. Later Geoffrion [7] defined proper efficiency by eliminating unbounded tradeoffs between the objectives and related it to Kuhn-Tucker proper efficiency. This concept was further generalised by Borwein [2] and Benson [1] with respect to domination sets which are closed convex cones. Borwein's proper efficiency is given in terms of contingent cone whereas Benson's proper efficiency is given in terms of projecting cone. According to Henig [8] a point is properly efficient if the decision set can be separated from the domination cone with origin in the point. Henig [8] considered the notion of global and local properness and established the equivalence of global (local) properness and Benson's (Borwein's) properness. In the same paper it is also shown that whenever the set of

Received 20th August, 2004

Copyright Clearance Centre, Inc. Serial-fee code: 0004-9727/05 \$A2.00+0.00. 
global proper efficient points is nonempty it is identical with the set of local proper efficient points. In one of the recent papers by Miettinen and Mäkelä [10], a characterisation of Henig's proper efficiency, with the nonnegative orthant as the domination set, is given in terms of normal cones of the feasible criterion set of a multiobjective optimisation problem.

The paper is organised as follows. Section 2 presents various notions of tangent and normal cones. In Section 3 we introduce a new notion of proper efficiency involving a proximal normal cone [5] and relate it with both Benson and Borwein proper efficiency. It is shown that proximal proper efficiency refines Borwein's proper efficiency and is independent of Benson's proper efficiency. In Section 4 we scalarise proximal proper efficiency for the case where the translation of the criterion set by the underlying cone is assumed to be locally star shaped; thus relaxing the convexity assumptions usually required to provide such characterisation (see [14]). It is also observed that scalar characterisation is not valid in the case of Benson proper efficiency under local star shapedness assumption. Section 5 deals with characterisation of Benson and Borwein proper efficiency in terms of normal cones assuming the convexity of a set related to the criterion set. It is also observed that a complete characterisation is not possible for the Benson proper efficiency for the nonconvex case.

\section{Preliminaries}

A set $C \subseteq R^{p}$ is said to be a cone if $\lambda c \in C$ for any $c \in C, \lambda \geqslant 0$ and $\lambda \in R$. A cone $C$ is said to be pointed if $C \cap(-C)=\{0\}$, and convex if $C+C \subseteq C$. The positive dual cone of a set $A \subseteq R^{p}$ is defined as

$$
A^{*}=\left\{d \in R^{p} \mid\langle d, a\rangle \geqslant 0, \forall a \in A\right\}
$$

and the strict positive dual cone of $\mathrm{A}$ is defined as

$$
A^{* 0}=\left\{d \in R^{p} \mid\langle d, a\rangle>0, \forall a \in A \backslash\{0\}\right\} .
$$

The cone generated by $A$, denoted by cone $A$, is defined as

$$
\operatorname{cone} A=\{\lambda a \mid \lambda \geqslant 0, \lambda \in R, a \in A\} .
$$

For further details refer to the book by Rockafellar [11]. The following implications follow immediately from the above definitions.

LEMMA 2.1. If $C$ is a pointed convex cone in $R^{p}$ then

(i) $(-C)^{*}=-C^{*}$

(ii) $C^{* 0}+C^{*} \subseteq C^{* 0}$.

LEMma 2.2. If $A$ and $B$ are subsets of $R^{p}$ such that $A \subseteq B$ then $B^{*} \subseteq A^{*}$. 
Several kinds of tangent cones have been considered by various authors in the literature. We now give the definition of two types of tangent cones.

Let $A$ be a set in $R^{p}$ and $\bar{a} \in A$. The contingent cone or Bouligand tangent cone to $A$ at $\bar{a}$, (see [12]), denoted by $T(A, \bar{a})$, is defined as

$$
T(A, \bar{a})=\left\{d \in R^{p} \mid \exists t_{j} \downarrow 0, d_{j} \rightarrow d \text { with } d_{j} \in A \text { such that } \bar{a}+t_{j} d_{j} \in A\right\} .
$$

The Clarke tangent cone to $A$ at $\bar{a}$, (Clarke [4]) denoted by $T_{C}(A, \bar{a})$ is defined as

$$
\begin{array}{r}
T_{C}(A, \bar{a})=\left\{d \in R^{p} \mid \forall t_{j} \downarrow 0, a_{j} \rightarrow \bar{a} \text { with } a_{j} \in A, \exists d_{j} \rightarrow d\right. \\
\left.\quad \text { such that } a_{j}+t_{j} d_{j} \in A\right\} .
\end{array}
$$

Both contingent and Clarke tangent cones are closed and

$$
T_{C}(A, \bar{a}) \subseteq T(A, \bar{a}) \subseteq \operatorname{cl} \operatorname{cone}(A-\bar{a})
$$

The tangent cone $T_{C}(A, \bar{a})$ is a convex cone whereas $T(A, \bar{a})$ is not necessarily convex. However if $A$ is a convex set then (see Borwein and Lewis [3, Corollary 6.3.7])

$$
T_{C}(A, \bar{a})=T(A, \bar{a})=\operatorname{cl} \operatorname{cone}(A-\bar{a}) .
$$

The notion of tangential regularity has been considered by Miettinen and Mäkelä [10] and Borwein and Lewis [3]. According to them a set $A$ is said to be tangentially regular at $\bar{a}$ if $T(A, \bar{a})=T_{C}(A, \bar{a})$. It is obvious that a convex set $A$ is tangentially regular at each of its points.

Normal cones play an important role in the field of optimisation, and have been introduced in various ways. One way of defining a normal cone is as the negative dual of the tangent cone. Accordingly the contingent normal cone to $A$ at $\bar{a}$, denoted by $N(A, \bar{a})$ is the negative dual of $T(A, \bar{a})$, that is

$$
N(A, \bar{a})=\left\{d \in R^{p} \mid\langle d, h\rangle \leqslant 0, \forall h \in T(A, \bar{a})\right\}
$$

and the Clarke normal cone to $A$ at $\bar{a}$, denoted by $N_{C}(A, \bar{a})$ is the negative dual of $T_{C}(A, \bar{a})$, that is

$$
N_{C}(A, \bar{a})=\left\{d \in R^{p} \mid\langle d, h\rangle \leqslant 0, \forall h \in T_{C}(A, \bar{a})\right\}
$$

From Lemma 2.1(i) it follows that

$$
N(A, \bar{a})=-T(A, \bar{a})^{*}
$$

and

$$
N_{C}(A, \bar{a})=-T_{C}(A, \bar{a})^{*}
$$


By Lemma 2.2 and relation (1) it follows that $N(A, \bar{a}) \subseteq N_{C}(A, \bar{a})$.

Proximal normals are direction vectors pointing outward from a set obtained by taking the projection of a point onto the set. The collection of all such perpendicular vectors at a point generates the proximal normal cone to the set at that point. We now give some notations and definitions from Clarke, Ledyaev, Stern and Wolenski [5]

Let $Y$ be a closed set in $R^{p}$ and $x$ be a point not lying in $Y$. Let $\bar{y}$ be projection of $x$ onto $Y$, that is,

$$
\|x-\bar{y}\|=\min _{u \in Y}\|x-u\| .
$$

The vector $x-\bar{y}$ is a proximal normal direction to $Y$ at $\bar{y}$ and any nonnegative multiple of such vector is called a proximal normal to $Y$ at $\bar{y}$. The set of all such vectors form the proximal normal cone to $Y$ at $\bar{y}$ and is denoted by $N_{P}(Y, \bar{y})$.

If $Y$ is a closed convex set then $N(Y, \bar{y}), N_{C}(Y, \bar{y})$ and $N_{P}(Y, \bar{y})$ coincide. Every proximal normal cone is a contingent normal cone, however the converse may not be necessarily true. For details and examples refer to Rockafellar and Wets [13]. Also we have $N_{C}(Y, \bar{y})=\overline{\mathrm{co}}\left\{\lim \xi_{i} \mid \xi_{i} \in N_{P}\left(Y, y_{i}\right), y_{i} \rightarrow \bar{y}\right\}$ where $\overline{c o}$ A denotes the closed convex hull of $A$ (see [5, Theorem 6.1]).

The following result gives a characterisation for the proximal normal vector in the form of an inequality.

LEMMA 2.3. ([5]) $A$ vector $\xi$ belongs to $N_{P}(Y, \bar{y})$ if and only if there exists $\sigma=\sigma(\xi, \bar{y}) \geqslant 0$ such that

$$
\langle\xi, y-\bar{y}\rangle \leqslant \sigma\|y-\bar{y}\|^{2} \quad \forall y \in Y .
$$

In particular if $Y$ is a convex set then

$$
\langle\xi, y-\bar{y}\rangle \leqslant 0 \quad \forall y \in Y .
$$

\section{Proximal Proper Efficiency}

Let $Y$ be a set in $R^{p}$ and $C$ be a closed convex pointed cone in $R^{p}$. A point $\bar{y} \in Y$ is said to be an efficient point of $Y$ if $(Y-\bar{y}) \cap(-C)=\{0\}$. A point $\bar{y} \in Y$ is said to be a Borwein proper efficient point (see [2]) of $Y$ if $T(Y+C, \bar{y}) \cap(-C)=\{0\}$ and Benson proper efficient point (see [11]) of $Y$ if $\operatorname{clcone}(Y+C-\bar{y}) \cap(-C)=\{0\}$.

We denote the set of efficient, Borwein proper efficient, Benson proper efficient solutions of $Y$ by $\operatorname{Eff}[Y, C], \operatorname{Bor}[Y, C], \operatorname{Ben}[Y, C]$, respectively. It can be seen that

$$
\operatorname{Ben}[Y, C] \subseteq \operatorname{Bor}[Y, C] \subseteq \operatorname{Eff}[Y, C]
$$

If $Y+C$ is a convex set then by (2) we have

$$
\operatorname{Ben}[Y, C]=\operatorname{Bor}[Y, C] \text {. }
$$


We now introduce the notion of proper efficiency in terms of the proximal normal cone and study its relation with Borwein and Benson proper efficiency

Definition 3.1: Let $Y$ be a closed set in $R^{p}$. A point $\bar{y} \in Y$ is called a proximal proper efficient point of $Y$ if

(i) $\bar{y} \in \operatorname{Eff}[Y, C]$

(ii) $N_{P}(Y+C, \bar{y}) \cap\left(-C^{* 0}\right) \neq \phi$.

The set of all proximal proper efficient points of $Y$ is denoted by $\operatorname{Pr}[Y, C]$.

The following theorem establishes that every proximal proper efficient point of a closed set $Y$ is a Borwein proper efficient point of $Y$.

TheOREM 3.1. For a closed subset $Y$ of $R^{p}, \operatorname{Pr}[Y, C] \subseteq \operatorname{Bor}[Y, C]$.

Proof: Let $\bar{y} \in \operatorname{Pr}[Y, C]$. By definition $(Y-\bar{y}) \cap(-C)=\{0\}$ and $N_{P}(Y+C, \bar{y})$ $\cap\left(-C^{* 0}\right) \neq \phi$. Let $h \in N_{P}(Y+C, \bar{y}) \cap\left(-C^{* 0}\right)$. Since $h \in N_{P}(Y+C, \bar{y})$, by Lemma 2.3, there exists $\sigma \geqslant 0$ such that

$$
\langle h, y-\bar{y}\rangle \leqslant \sigma\|y-\bar{y}\|^{2} \quad \forall y \in Y+C .
$$

If $\bar{y} \notin \operatorname{Bor}[Y, C]$ then there exists $d \neq 0$ such that $d \in T(Y+C, \bar{y}) \cap(-C)$. As $d \in T(Y+C, \bar{y})$, there exist $t_{j} \downarrow 0, y_{j} \in Y, c_{j} \in C$ with $y_{j}+c_{j} \rightarrow d$ such that $\bar{y}+t_{j}\left(y_{j}+c_{j}\right) \in Y+C$. Hence from (5) it follows that

$$
\left\langle h, t_{j}\left(y_{j}+c_{j}\right)\right\rangle \leqslant \sigma\left\|t_{j}\left(y_{j}+c_{j}\right)\right\|^{2} .
$$

Assuming $t_{j}>0$, we have

$$
\left\langle h, y_{j}+c_{j}\right\rangle \leqslant \sigma t_{j}\left\|y_{j}+c_{j}\right\|^{2}
$$

which on taking limit $j \rightarrow \infty$ implies $\langle h, d\rangle \leqslant 0$. Also since $d \in-C \backslash\{0\}$ and $h \in-C^{* 0}$ we have $\langle h, d\rangle>0$ which is a contradiction. Hence it follows that $\bar{y} \in \operatorname{Bor}[Y, C]$.

We now give examples to illustrate that a Benson proper efficient point is not necessarily a proximal proper efficient point and vice versa.

EXAMPLE 3.1. Let $C=R_{+}^{2}$ and let $Y=\left\{\left(y_{1}, y_{2}\right) \mid 2 y_{2} \geqslant-y_{1}\right\} \cup\left\{\left(y_{1}, y_{2}\right) \mid y_{2} \geqslant-2 y_{1}\right\}$. It can be seen that $\bar{y}=(0,0) \in \operatorname{Ben}[Y, C]$ as cl cone $(Y+C-\bar{y})=Y$ and $Y \cap(-C)=\{0\}$. However $\bar{y} \notin \operatorname{Pr}[Y, C]$ as $N_{P}(Y+C, \bar{y})=\{0\}$.

REMARK 3.1. As $\operatorname{Ben}[Y, C] \subseteq \operatorname{Bor}[Y, C]$ we have in the above example $\bar{y}=(0,0)$ $\in \operatorname{Bor}[Y, C]$ and $\bar{y} \notin \operatorname{Pr}[Y, C]$ establishing that $\operatorname{Bor}[Y, C] \not \subset \operatorname{Pr}[Y, C]$.

ExAmple 3.2. Let $C=R_{+}^{2}$ and let $Y=\left\{\left(y_{1}, y_{2}\right) \mid y_{1}<0, y_{2} \geqslant 1\right\} \cup R_{+}^{2}$. Since for $\bar{y}=(0,0), \operatorname{cl}$ cone $(Y+C-\bar{y})=\left\{\left(y_{1}, y_{2}\right) \mid y_{2} \geqslant 0\right\}$ and cl cone $(Y+C-\bar{y}) \cap(-C)$ $=\left\{\left(y_{1}, y_{2}\right) \mid y_{1} \leqslant 0, y_{2}=0\right\} \neq\{0\}$ the point $\bar{y} \notin \operatorname{Ben}[Y, C]$. Clearly $-C^{* 0}=\left\{\left(y_{1}, y_{2}\right) \mid\right.$ $\left.y_{1}<0, y_{2}<0\right\}$ and $N_{P}(Y+C, \bar{y})=-C$, hence $\bar{y} \in \operatorname{Pr}[Y, C]$.

Figure 1 summarises the result so far. 


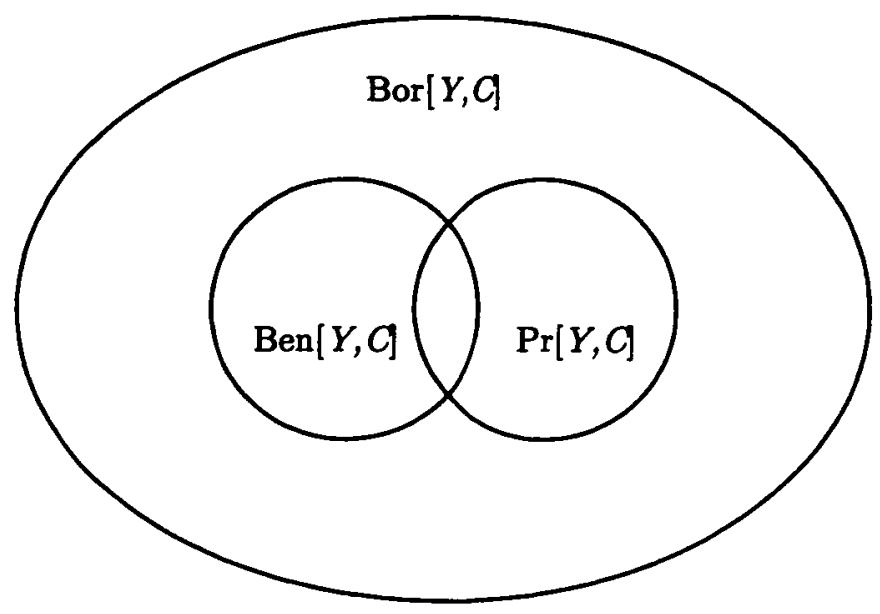

Figure 1: Relation among Benson, Borwein and proximal proper efficient points

\section{Scalarisation}

We now scalarise proximal proper efficient points by vectors in the strict dual cone of $C$. For this purpose we require the following notations as given in Sawaragi, Nakayama and Tanino [14, Section 3.4]. Let

$$
S_{\mu} Y=\{\bar{y} \mid\langle\mu, \bar{y}\rangle=\inf \{\langle\mu, y\rangle \mid y \in Y\}\} .
$$

Geometrically $S_{\mu} Y$ is the set of supporting points of $Y$ with the inner normal vector $\mu$. It can be seen that $S_{\alpha \mu} Y=S_{\mu} Y$ for $\alpha>0$. Let

$$
D[Y, C]=\bigcup_{\mu \in C^{* 0}} S_{\mu} Y .
$$

We now investigate the relation between $D[Y, C]$ and $\operatorname{Pr}[Y, C]$.

TheOREM 4.1. For a closed set $Y$ in $R^{p}, D[Y, C] \subseteq \operatorname{Pr}[Y, C]$.

PRoOF: Let $\bar{y} \in D[Y, C]$. Then there exists $\mu \in C^{* 0}$ such that $\bar{y} \in S_{\mu} Y$, that is,

$$
\langle\mu, \bar{y}\rangle \leqslant\langle\mu, y\rangle \quad \forall y \in Y .
$$

Let if possible, $\bar{y} \notin \operatorname{Eff}[Y, C]$. Then there exists $d \neq 0$ such that $d \in(Y-\bar{y}) \cap(-C)$, that is, there exists $y \in Y$ such that $d=y-\bar{y}$ and $d \in-C$. As $\mu \in C^{* 0}$ we have $\langle\mu, d\rangle<0$, that is $\langle\mu, y-\bar{y}\rangle<0$ which contradicts (6). Hence $\bar{y} \in \operatorname{Eff}[Y, C]$. We shall now show that $-\mu \in N_{P}(Y+C, \bar{y})$. For any $c \in C$ we have $\langle-\mu, c\rangle \leqslant 0$ which together with (6) yields

$$
\langle-\mu, y+c-\bar{y}\rangle \leqslant 0 \quad \forall y \in Y .
$$

Hence $-\mu \in N_{P}(Y+C, \bar{y})$. Thus, $\bar{y} \in \operatorname{Pr}[Y, C]$ as $-\mu \in N_{P}(Y+C, \bar{y}) \cap\left(-C^{\star 0}\right)$. 
REMARK 4.1. In [14] it has been established that if $C$ is a closed convex cone in $R^{p}$ then $D[Y, C] \subseteq \operatorname{Ben}[Y, C]$. By (4) it is also obvious that $D[Y, C] \subseteq \operatorname{Bor}[Y, C]$.

We now have the notion of local star shapedness introduced by Ewing [6]. A set $A$ in $R^{p}$ is said to be locally star shaped at $x^{*} \in A$ if for any $x \in A$, there exists $a\left(x, x^{*}\right)$ where $0<a\left(x, x^{*}\right) \leqslant 1$ such that $(1-\lambda) x^{*}+\lambda x \in A$ for $0<\lambda<a\left(x, x^{*}\right) . A$ is said to be locally star shaped if it is locally star shaped at each of its points. From the definition it is obvious that if $a\left(x, x^{*}\right)=1$ for every $x, x^{*} \in A$ then the set $A$ is a convex set. Thus every convex set is locally star shaped but the converse may not necessarily be true. A simple example of a locally star shaped set which is not convex is the set of union of two disjoint open intervals in $R$.

A set $A$ is said to be a $C$-convex set (see [14, Definition 2.1.9]) if $A+C$ is a convex set. Clearly, as observed above, if $A$ is a $C$-convex set then $A+C$ is a locally star shaped set.

THEOREM 4.2. For a closed set $Y$ in $R^{p}$ if $\bar{y} \in \operatorname{Pr}[Y, C]$ and $Y+C$ is locally star shaped at $\bar{y}$, then $\bar{y} \in D[Y, C]$.

Proof: Since $\bar{y} \in \operatorname{Pr}[Y, C]$, there exists $h \in-C^{* 0}$ such that $h \in N_{P}(Y+C, \bar{y})$. By proximal normal inequality there exists $\sigma \geqslant 0$ such that

$$
\langle h, \widehat{y}-\bar{y}\rangle \leqslant \sigma\|\widehat{y}-\bar{y}\|^{2} \quad \forall \hat{y} \in Y+C .
$$

As $Y+C$ is locally star shaped at $\bar{y}$, for any $y \in Y$, there exists $a(y, \bar{y})$ where $0<a(y, \bar{y}) \leqslant 1$ such that $\bar{y}+\lambda(y-\bar{y}) \in Y+C$ for $0<\lambda<a(y, \bar{y})$. Hence from (7) it is obvious that

$$
\langle h, \lambda(y-\bar{y})\rangle \leqslant \sigma \lambda^{2}\|y-\bar{y}\|^{2} \quad \forall y \in Y .
$$

As $\lambda>0$, we get

$$
\langle h, y-\bar{y}\rangle \leqslant \sigma \lambda\|y-\bar{y}\|^{2} \quad \forall y \in Y .
$$

On taking limit $\lambda \rightarrow 0+$, we have for every $y \in Y,\langle h, y-\bar{y}\rangle \leqslant 0$. Let $\mu=-h$, then $\mu \in C^{* 0}$ and $\langle\mu, \bar{y}\rangle \leqslant\langle\mu, y\rangle, \forall y \in Y$, that is $\bar{y} \in S_{\mu} Y$. Since $\mu \in C^{* 0}$ it follows that $\bar{y} \in D[Y, C]$.

The following example illustrates that the above theorem does not hold in the absence of local star shapedness.

EXAMPLE 4.1. Let $C=R_{+}^{2}$ and $Y=\left\{\left(y_{1}, y_{2}\right) \mid y_{2}=-y_{1}, 0 \leqslant y_{1} \leqslant 2\right\} \cup\left\{\left(y_{1}, y_{2}\right) \mid\right.$ $\left.y_{2}=2,-2 \leqslant y_{1}<0\right\}$. It can be seen that $Y+C$ is not locally star shaped at $\bar{y}=(0,0)$ as $(1-\lambda) \bar{y}+\lambda y \notin Y+C$ for $0<\lambda<1$ where $y=(-1,2)$. As $N_{P}(Y+C, \bar{y})=-C$ it follows that $\bar{y} \in \operatorname{Pr}[Y, C]$. Now $C^{* 0}=\left\{\left(\mu_{1}, \mu_{2}\right) \mid \mu_{1}>0, \mu_{2}>0\right\}$ and for $\mu=\left(\mu_{1}, \mu_{2}\right) \in C^{* 0}$

$$
S_{\mu} Y= \begin{cases}\{(-2,2)\} & \text { if } \mu_{1} \geqslant \mu_{2} \\ \{(2,-2)\} & \text { if } \mu_{1}<\mu_{2} .\end{cases}
$$


Thus $D[Y, C]=\{(-2,2),(2,-2)\}$ and hence $\bar{y} \notin D[Y, C]$.

REMARK 4.2. In Sawaragi, Nakayama and Tanino [14] it has been shown that if $C$ is a closed convex pointed cone and $Y$ is a $C$-convex set, then $\operatorname{Ben}[Y, C] \subseteq D[Y, C]$. As $\operatorname{Ben}[Y, C]=\operatorname{Bor}[Y, C]$, if $Y+C$ is a convex set, it follows that $\operatorname{Bor}[Y, C] \subseteq D[Y, C]$ if $C$ is a closed convex pointed cone in $Y$ and $Y$ is a $C$-convex set.

We now give an example to illustrate $C$-convexity cannot be replaced by local star shapedness for Benson proper efficient points.

EXAMPLE 4.2. Let $C=R_{+}^{2}$ and $Y=\left\{\left(y_{1}, y_{2}\right) \mid y_{2}=-y_{1},-2 \leqslant y_{1}<0\right\} \cup\left\{\left(y_{1}, y_{2}\right) \mid\right.$ $\left.y_{2}=-2 y_{1}, 0 \leqslant y_{1} \leqslant 2\right\}$. Note that $Y+C$ is a locally star shaped at $\bar{y}=(0,0)$ but $Y$ is not a $C$-convex set. Also

$$
\begin{aligned}
\operatorname{cl} \text { cone }(Y+C-\bar{y})=T(Y+C, \bar{y})=\left\{\left(y_{1}, y_{2}\right) \mid y_{2}\right. & \left.\geqslant-y_{1},-2 \leqslant y_{1}<0\right\} \\
& \cup\left\{\left(y_{1}, y_{2}\right) \mid y_{2} \geqslant-2 y_{1}, 0 \leqslant y_{1} \leqslant 2\right\} .
\end{aligned}
$$

Hence $\bar{y} \in \operatorname{Ben}[Y, C](\operatorname{Bor}[Y, C])$ as cl cone $(Y+C-\bar{y}) \cap(-C)=\{0\}$. For $\mu=\left(\mu_{1}, \mu_{2}\right)$ $\in C^{* 0}$

$$
S_{\mu} Y= \begin{cases}\{(-2,2)\} & \text { if } \mu_{1} \geqslant 2 \mu_{2} \text { or } 2 \mu_{2}>\mu_{1} \geqslant(3 / 2) \mu_{2} \\ \{(2,-4)\} & \text { if }(3 / 2) \mu_{2}>\mu_{1} \geqslant \mu_{2} \text { or } \mu_{1}<\mu_{2} .\end{cases}
$$

so we have $D[Y, C]=\{(-2,2),(2,-4)\}$ and hence $\bar{y} \notin D[Y, C]$. In this example it may also be seen that $\bar{y} \notin \operatorname{Pr}[Y, C]$ as $N_{P}(Y+C, \bar{y})=\{0\}$.

\section{Characterisation of Benson and Borwein Proper efFiciency in terms} OF NORMAL CONES

Recently Miettinen and Mäkelä [10] characterised proper efficiency in the sense of Henig [8], for a multiobjective optimisation problem with the help of normal cones for both the cases when the feasible criterion set is convex as well as nonconvex (see [8, Theorem 4 and Theorem 9]). Motivated by this result, in this section, we give similar characterisation for Benson and Borwein proper efficient points in terms of normal cones. We first discuss the case when $Y+C$ is a convex set where $Y \subseteq R^{p}$ and $C$ is a convex pointed cone in $R^{p}$. We require the following lemma which follows from [11, Corollary 16.4.2] where the result is given for negative dual cone.

LEMma 5.1. Let $C_{1}, C_{2}, \ldots, C_{m}$ be nonempty convex cones in $R^{p}$. Then

$$
\left(\mathrm{cl} C_{1} \cap \mathrm{cl} C_{2} \cap \ldots \cap \mathrm{cl} C_{m}\right)^{*}=\operatorname{cl}\left(C_{1}^{*}+C_{2}^{*}+\ldots+C_{m}^{*}\right) \text {. }
$$

THEOREM 5.1. Let $Y+C$ be a convex set in $R^{p}$. Then $\bar{y} \in \operatorname{Bor}[Y, C](\operatorname{Ben}[Y, C])$ if and only if $N(Y+C, \bar{y}) \cap\left(-C^{* 0}\right) \neq \phi$. 
Proof: If $\bar{y} \in \operatorname{Bor}[Y, C]$ then by definition we have $T(Y+C, \bar{y}) \cap(-C)=\{0\}$ which implies $(T(Y+C, \bar{y}) \cap(-C))^{*}=(\{0\})^{*}$. Using Lemma 5.1 and (3) we have $-N(Y+C, \bar{y})+(-C)^{*}=R^{p}$. Now by Lemma 2.1 (i) it follows that

$$
-N(Y+C, \bar{y})-C^{*}=R^{p}
$$

Let $x \in C^{* 0} \subseteq R^{p}$ then by (8) there exists $h \in N(Y+C, \bar{y}), c \in C^{*}$ such that $-h-c=x$. Using Lemma 2.1 (ii) we have $-h=x+c \in C^{* 0}+C^{*} \subseteq C^{* 0}$. Hence $h \in N(Y+C, \bar{y})$ $\cap\left(-C^{* 0}\right)$ that is $N(Y+C, \bar{y}) \cap\left(-C^{* 0}\right) \neq \phi$.

Conversely, let $N(Y+C, \bar{y}) \cap\left(-C^{* 0}\right) \neq \phi$. Let $h \in N(Y+C, \bar{y}) \cap\left(-C^{* 0}\right)$. Let us suppose that $\bar{y} \notin \operatorname{Bor}[Y, C]$, that is there exists $d \neq 0$ such that $d \in T(Y+C, \bar{y}) \cap(-C)$. As $h \in N(Y+C, \bar{y})$ and $d \in T(Y+C, \bar{y})$ we have $\langle h, d\rangle \leqslant 0$. Also since $d \in-C \backslash\{0\}$ and $h \in-C^{* 0}$ it follows that $\langle h, d\rangle>0$ which is a contradiction. Hence it follows that $\bar{y} \in \operatorname{Bor}[Y, C]$. The proof is complete since $\operatorname{Ben}[Y, C]=\operatorname{Bor}[Y, C]$ when $Y+C$ is a convex set.

We now generalise the above result when $Y+C$ is no longer a convex set.

Theorem 5.2. If $\bar{y} \in \operatorname{Bor}[Y, C](\operatorname{Ben}[Y, C])$ then $N_{C}(Y+C, \bar{y}) \cap\left(-C^{* 0}\right) \neq \phi$.

Proof: If $\bar{y} \in \operatorname{Bor}[Y, C](\operatorname{Ben}[Y, C])$ then $T(Y+C, \bar{y}) \cap(-C)=\{0\}$ (clcone $(Y+C-\bar{y}) \cap(-C)=\{0\})$. Using the relation (2) we have $T_{C}(Y+C, \bar{y}) \cap(-C)=\{0\}$. Proceeding as in Theorem 5.1 we get the required result.

THEOREM 5.3. If $Y+C$ is tangentially regular at $\bar{y}$ and $N_{C}(Y+C, \bar{y}) \cap\left(-C^{* 0}\right)$ $\neq \phi$ then $\bar{y} \in \operatorname{Bor}[Y, C]$.

Proof: If $Y+C$ is tangentially regular at $\bar{y}$, then $(T(Y+C, \bar{y}))^{*}=\left(T_{C}(Y+C, \bar{y})\right)^{*}$ that is, $N(Y+C, \bar{y})=N_{C}(Y+C, \bar{y})$. By the given hypothesis we have $N(Y+C, \bar{y})$ $\cap\left(-C^{* 0}\right) \neq \phi$. Rest of the proof follows as in the converse part of Theorem 5.1.

The following example illustrates that the above theorem may not necessarily hold for Benson proper efficient point even if $Y+C$ is tangentially regular.

Example 5.1. Consider the set $C$ and $Y$ considered in Example 3.2. It was observed that $\bar{y}=(0,0) \notin \operatorname{Ben}[Y, C]$. Also $Y$ is tangentially regular at $\bar{y}$ as $T(Y+C, \bar{y})$ $=T_{C}(Y+C, \bar{y})=R_{+}^{2}$. As $N(Y+C, \bar{y})=-C$ we have that $N(Y+C, \bar{y}) \cap\left(-C^{* 0}\right) \neq \phi$. Since $T(Y+C, \bar{y}) \cap(-C)=\{0\}$ it follows that $\bar{y} \in \operatorname{Bor}[Y, C]$.

We thus observe that a complete characterisation is not available for Benson proper efficiency when $Y+C$ is a nonconvex set.

\section{Conclusions}

One of the major advantages of proximal proper efficiency is that a scalar characterisation is possible even when the criterion set is not necessarily convex. Unlike the 
case with Benson and Borwein proper efficiency we only require the translation of the criterion set by the underlying cone to be locally star shaped. The geometric nature of proximal normal cone facilitates the identification of the improper solutions. However the only limitation with proximal proper efficiency in comparison with the other similar notions, is that, the underlying set to has to be a closed set.

\section{REFERENCES}

[1] H.P. Benson, 'An improved version of proper efficiency for vector minimization with respect to cones', J. Math. Anal. Appl. 71 (1979), 232-241.

[2] J.M. Borwein, 'Proper efficient points for maximization with respect to cones', SIAM J. Control Optim. 15 (1977), 57-63.

[3] J.M. Borwein and A.S. Lewis, Convex analysis and nonlinear optimization: Theory and examples (Springer Verlag, New York, 2000).

[4] F.H. Clarke, Optimization and nonsmooth analysis (John Wiley and Sons, New York, 1970).

[5] F.H. Clarke, Y.S. Ledyaev, R.J. Stern and P.R. Wolenski, Nonsmooth analysis and control theory (Springer Verlag, New York, 1998).

[6] G.M. Ewing, 'Sufficient conditions for global minima of suitable concave functionals from variational and control theory', SIAM Rev. 19 (1977), 202-220.

[7] A.M. Geoffiion, 'Proper efficiency and the theory of vector maximization', J. Math. Anal. Appl. 22 (1968), 618-630.

[8] M.I. Henig, 'Existence and characterization of efficient decisions with respect to cones', Math. Prog. 23 (1982), 111-116.

[9] H.W. Kuhn and A.W. Tucker, 'Nonlinear programming', in Proceedings of the Second Berkeley Symposium on Mathematical Statistics and Probability (University of California Press, Berkeley, CA, 1951), pp. 481-492.

[10] K. Miettinen and M.M. Mäkelä, 'On cone characterizations of weak, proper and pareto optimality in multiobjective optimization', Math. Methods Oper. Res. 53 (2001), 233-245.

[11] R.T. Rockafellar, Convex analysis (Princeton University Press, Princeton, N.J., 1970).

[12] R.T. Rockafellar, The theory of subgradients and its applications to problems of optimization: convex and nonconvex functions (Heldermann Verlag, Berlin, 1981).

[13] R.T. Rockafellar and R.J.-B. Wets, Variational analysis (Springer Verlag, Berlin, Heidelberg, 1998).

[14] Y. Sawaragi, H. Nakayama and T. Tanino, Theory of multiobjective optimization (Academic Press, Orlando, FL, 1985).

Department of Mathematics

Rajdhani College

University of Delhi

Raja Garden

New Delhi-110 015

India

e-mail: cslalitha@rediffmail.com

\author{
Department of Mathematics \\ University of Delhi \\ Delhi - 110007 \\ India \\ e-mail: ruchiiq@yahoo.co.in
}

